\title{
Study of scintillation counter consisting of a pure CsI crystal and avalanche photodiodes
}

\author{
H. Aihara \\ The University of Tokyo, 7-3-1 Hongo Bunkyo-ku, Tokyo 113-0033, Japan \\ O. V. Borshchev \\ Enikolopov Institute of Synthetic Polymeric Materials of the Russian Academy of Sciences, \\ Profsoyuznaya st. 70, Moscow 117393, Russia
}

\section{A. Epifanov*}

The University of Tokyo, 7-3-1 Hongo Bunkyo-ku, Tokyo 113-0033, Japan

E-mail: epifanov@hep.phys.s.u-tokyo.ac.jp

Y. Jin

The University of Tokyo, 7-3-1 Hongo Bunkyo-ku, Tokyo 113-0033, Japan

\section{S. A. Ponomarenko}

Enikolopov Institute of Synthetic Polymeric Materials of the Russian Academy of Sciences, Profsoyuznaya st. 70, Moscow 117393, Russia

\section{N. M. Surin}

Enikolopov Institute of Synthetic Polymeric Materials of the Russian Academy of Sciences, Profsoyuznaya st. 70, Moscow 117393, Russia

The Belle II detector at the SuperKEKB $e^{+} e^{-}$collider is the only Super Flavor Factory in the nearest future. Electromagnetic calorimeter (ECL) is one of the most important subsystems of Belle II. To cope with extremely high luminosity and severe background conditions it was decided to upgrade the end cap ECL and utilize pure CsI scintillation crystals. We report about development and study of the counter based on the pure CsI crystal and avalanche photodiodes (of Hamamatsu S8664 series).

PACS: $29.40 . \mathrm{CS}, 29.40 . \mathrm{GX}$

International Conference on New Photo-detectors, PhotoDet 2015

6-9 July 2015

Moscow, Troitsk, Russia

${ }^{*}$ Speaker. 


\section{Introduction}

Successful asymmetric energy $e^{+} e^{-}$B Factories, Belle and $B A B A R$, raised lots of new intriguing questions in the flavor sector of the Standard Model. They confirmed the fruitfulness of the B Factory experimental strategy in the precision tests of the Standard Model and search for New Physics [1].

The Belle II detector at the SuperKEKB collider, successor of Belle at KEKB [2, 3], is the only $e^{+} e^{-}$Super Flavor Factory in the nearest future. It is competitive/complementary to the current and coming energy/intensity frontier experiments [4]. The expected instantaneous luminosity of $8 \times$ $10^{35} 1 / \mathrm{cm}^{2} / \mathrm{s}$ and the statistics of $50 \mathrm{ab}^{-1}$ represent great challenge to both SuperKEKB accelerator and Belle II detector [5]. To cope with extremely high luminosity and severe background conditions it was decided to upgrade the end cap electromagnetic calorimeter (ECL) and utilize fast pure CsI scintillation crystals with the scintillation decay time of about $30 \mathrm{~ns}$.

Two types of the photosensitive elements, vacuum photopentodes and silicon avalanche photodiodes (APDs), are considered. The second option with APD(s) (Hamamatsu S8664-55 and S8664-1010) is studied in this paper. Hamamatsu APD is a compact photosensor insensitive to magnetic field. The recommended operating point at the reverse bias voltage of about $400 \mathrm{~V}$, provides the gain of about 50 and dark current of few nanoamperes (at the temperature $T=25^{\circ} \mathrm{C}$ ).

High equivalent noise charge (ENC) originates from large junction capacitance of APD (100$300 \mathrm{pF}$ ) and necessarily short shaping time (30-50 ns). Taking into account modest light yield of pure CsI crystal $(2000 \div 5000$ photons/MeV) we get notable equivalent noise energy (ENE). In the studies of the counter based on the CsI(pure) crystal (of $6 \times 6 \times 30 \mathrm{~cm}^{3}$ size) and one APD Hamamatsu S8664-1010, the equivalent noise energy was measured to be $\mathrm{ENE} \simeq 2 \mathrm{MeV}$, which essentially exceeds the acceptable level $(\lesssim 0.5 \mathrm{MeV})[5]$.

\section{Characteristics of APD, measurement of equivalent noise charge}

The main characteristics of APD, dark current and gain as functions of bias voltage were measured at different temperatures. Figure 1 shows Hamamatsu S8664-1010 APD gain $(G)$ vs. bias voltage $\left(U_{\text {bias }}\right)$ for the temperatures from $10{ }^{\circ} \mathrm{C}$ up to $43{ }^{\circ} \mathrm{C}$ in the vicinity of the operating point $\left(U_{\text {bias }}=394 \mathrm{~V}, G=50, T=24^{\circ} \mathrm{C}\right.$ ). At the operating point the dark current of the S86641010 [S8664-55] APD varies from $1 \mathrm{nA}[1 \mathrm{nA}]$ up to $30 \mathrm{nA}[8 \mathrm{nA}]$ at the temperatures from $10{ }^{\circ} \mathrm{C}$ to $43{ }^{\circ} \mathrm{C}$. The relative temperature gain variations $\left(\frac{1}{G} \frac{d G}{d T}\right)$ for Hamamatsu S8664-1010 [S866455] APDs at different operating points with gain of 30, 50 and 100 were measured to be $(-2.3 \pm$ $0.1) \% /{ }^{\circ} \mathrm{C}\left[(-2.4 \pm 0.1) \% /{ }^{\circ} \mathrm{C}\right],(-3.1 \pm 0.1) \% /{ }^{\circ} \mathrm{C}\left[(-3.3 \pm 0.1) \% /{ }^{\circ} \mathrm{C}\right]$ and $(-4.9 \pm 0.2) \% /{ }^{\circ} \mathrm{C}$ $\left[(-5.0 \pm 0.2) \% /{ }^{\circ} \mathrm{C}\right]$, respectively. So, APD is a dominant source of the temperature variations of the signal from the counter based on pure CsI crystal and APD. To provide stable response, these variations can be compensated by temperature adjustment of the bias voltage. From Fig. 1 it is seen that the absolute [relative] temperature bias voltage variation to keep constant gain $G=50$ is $\frac{d U}{d T}=0.67 \mathrm{~V} /{ }^{\circ} \mathrm{C}\left[\frac{1}{U} \frac{d U}{d T}=0.17 \% /{ }^{\circ} \mathrm{C}\right]$. Such a fine tuning of the bias voltage can be implemented just on the preamplifier board with the temperature sensor mounted in the vicinity of APD.

The electronic readout chain is shown in Fig. 2. The ENC in the chain was studied with help of the electronic calibration signal. Calibration pulses with constant amplitude, $U_{0}$, are fed to the 

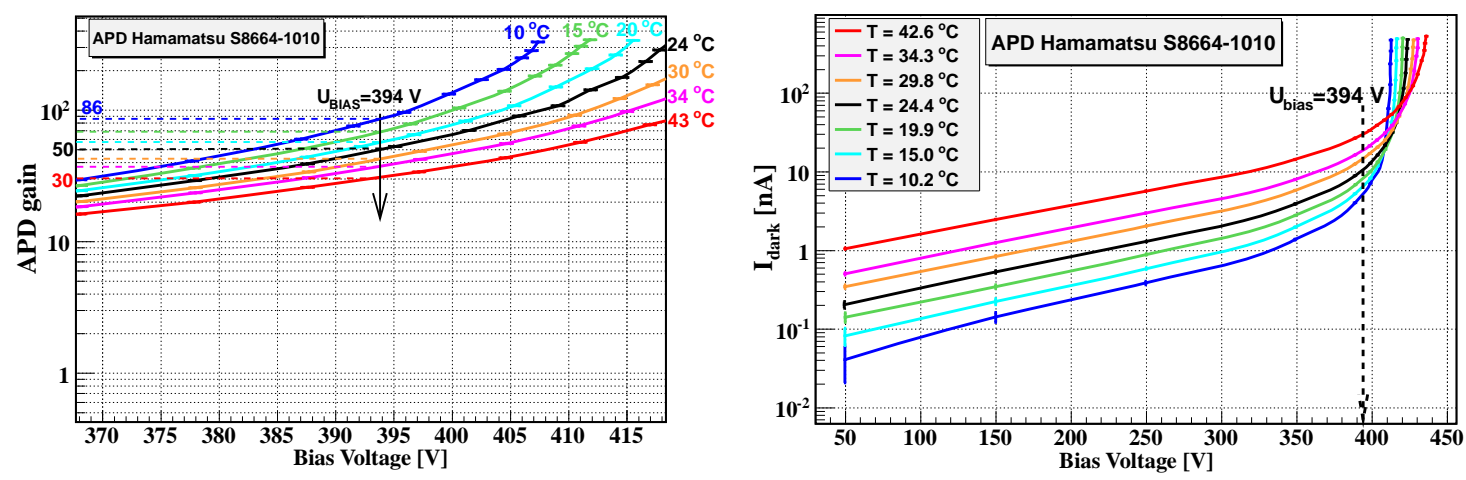

Figure 1: Hamamatsu S8664-1010 APD gain vs. bias voltage (left), dark current vs. bias voltage (right) at the temperatures $T=10^{\circ} \mathrm{C} \div 43{ }^{\circ} \mathrm{C}$.

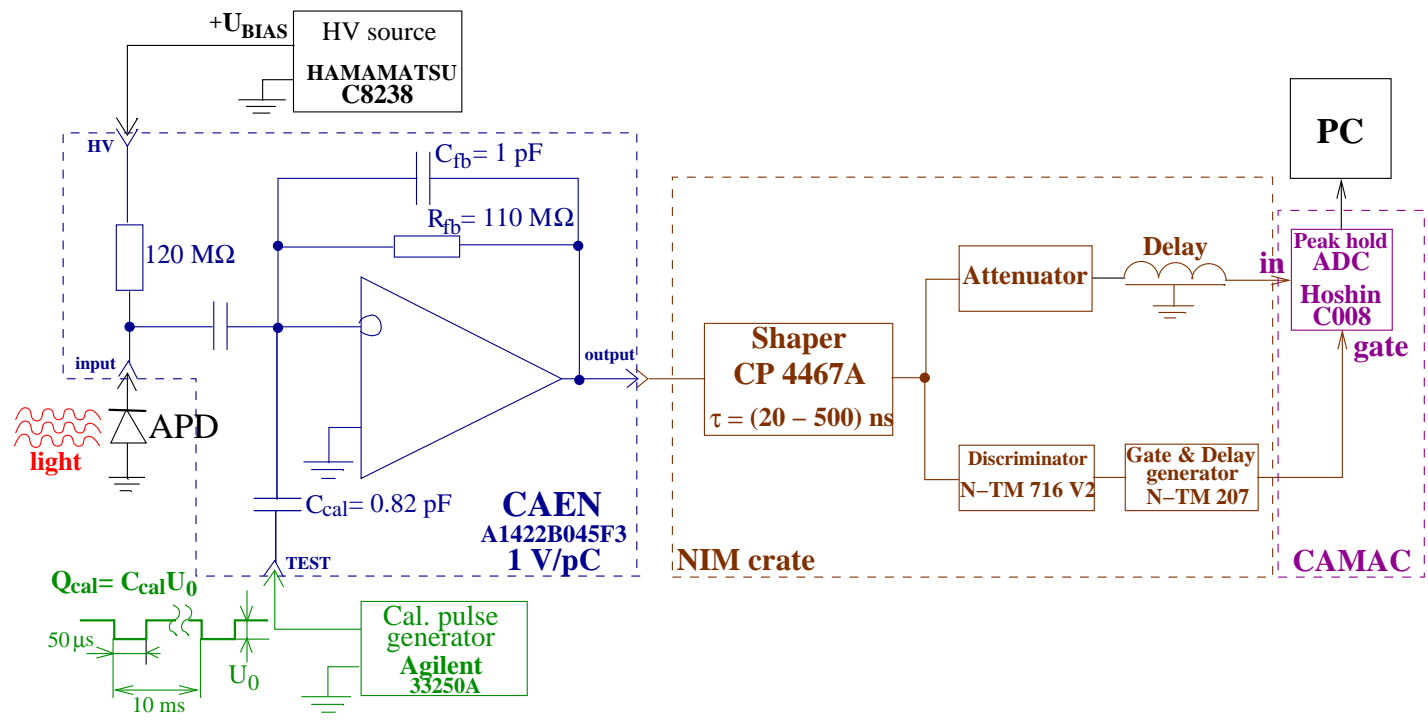

Figure 2: Block diagram of experimental setup for analyzing signal from APD.

test input of the charge sensitive preamplifier (CAEN A1422B045F3) producing incoming charge $Q_{\text {cal }}=C_{\text {cal }} U_{0}$, where $C_{\text {cal }}=(0.82 \pm 0.05) \mathrm{pF}$ is calibration capacitor. The output voltage from the preamplifier has an amplitude $U_{\text {out }}=Q_{\mathrm{cal}} / C_{\mathrm{fb}}$, where $C_{\mathrm{fb}}$ is feedback capacitor of preamplifier. Voltage signal from preamplifier is then filtered and amplified by shaper (Clear Pulse 4467A) and digitized by peak hold ADC (Hoshin C008). The position of the peak (mean value) in the amplitude spectrum is used to graduate ADC channels in the units of incoming charge, and the root mean square (r.m.s.) is a measure of the ENC (we evaluate ENC in the units of electron charge). The ENC squared in the spectrometric channel is [6]:

$$
E N C^{2}=A^{2} \tau+\left(\frac{B^{2}}{\tau}+E^{2}\right) C_{A P D}^{2}+D^{2},
$$

where $A=\sqrt{\frac{2 I_{d} g F K_{1}}{|e|}}$ is shot noise coefficient, $B=\frac{\sqrt{4 k_{B} T R_{s} K_{2}}}{|e|}$ is thermal noise coefficient, $E$ is $1 / \mathrm{f}$ noise factor and $D$ is additional noise produced in the secondary cascades of the preamplifier as well as by shaper and ADC. Here $e$ is electron charge, $I_{d}$ is the dark current in APD, $g$ is the 
gain of APD, $F$ is excess noise factor of APD, $k_{B}$ is Boltzmann constant, $T$ is the temperature of $\mathrm{APD}, R_{S}$ is equivalent series resistance, $C_{\mathrm{APD}}$ is junction capacitance of $\mathrm{APD}, \tau$ is shaping time, $K_{1}, K_{2}$ are the factors determined by the filtering scheme of the shaping amplifier. The equivalent series resistance, $R_{S}$, is determined mostly by the reversal transconductance of the input field effect transistor(s) (FET(s)) in the preamplifier (two BF862 FETs in CAEN A1422B045F3).

The graphs of ENC vs. shaping time in the spectrometric channels without APD (additional noise), with one APD S8664-55 and with one APD S8664-1010 are shown in Fig. 3.

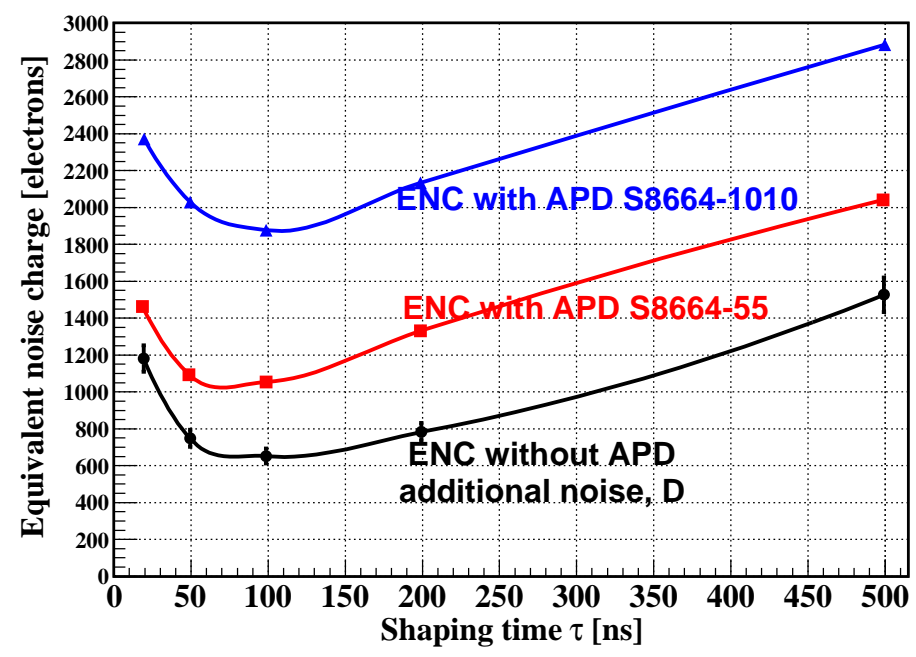

Figure 3: The ENC vs. shaping time in the spectrometric channels without APD (black points), with one APD S8664-55 (red points), with one APD S8664-1010 (blue points).

Each ENC component in Eq.2.1 was studied separately. To calculate shot noise (the first term in Eq.2.1) in addition to the dark current and gain we measured $F$ and $K_{1}$. The signal shape factor $K_{1}=\frac{\left.|e| \mid \mathrm{ENC}_{\text {photo }}^{2}-\mathrm{ENC}^{2}\right]}{2 I_{\text {photo }} \tau}=0.65 \pm 0.04$ was measured with PIN photodiode Hamamatsu S2744-08 instead of APD in the electronic chain. For the measurement of F, we utilized ORTEC 570 shaping amplifier with $K_{1}^{\prime}=0.44 \pm 0.02$, which allows us to set larger shaping times $(\tau=(0.5 \div 10) \mu \mathrm{s})$. The excess noise factor is measured with APD in the electronic chain: $F=\frac{|e|\left[\mathrm{ENC}_{\text {photo }}^{2}-\mathrm{ENC}^{2}\right]}{2 I_{\text {photo }} \tau g K_{1}^{\prime}}$. $\mathrm{ENC}_{\text {photo }}^{2}$ is the equivalent noise charge with the additional photocurrent, $I_{\text {photo }}$, when PIN photodiode/APD was illuminated by light-emitting diode. At the operating point the excess noise factor of the S8664 APD was measured to be $F=4.7 \pm 0.5$.

The thermal and 1/f noise components (the second term in Eq.2.1), proportional to $C_{\mathrm{APD}}$, were measured simultaneously. The ratio $\frac{\mathrm{ENC}_{2}^{2}-\mathrm{ENC}_{1}^{2}}{C_{2}^{2}-C_{1}^{2}}$ was measured at different shaping times $\tau=(20 \div 500) \mathrm{ns}$, where the $\mathrm{ENC}_{1,2}$ is equivalent noise charge with the $C_{1,2}$ test capacitor $\left(C_{1}=\right.$ $96 \mathrm{pF}, C_{2}=218 \mathrm{pF}$ ) at the preamplifier input instead of APD. It was approximated by the function, $\frac{B^{2}}{\tau}+E^{2}$, and the thermal noise coefficient $B=(26.2 \pm 0.8 \pm 4.8) \sqrt{\mathrm{ns}} / \mathrm{pF}$ (at the temperature $T=$ $\left.24{ }^{\circ} \mathrm{C}\right)$ and $1 / \mathrm{f}$ noise factor $E=(6.1 \pm 0.1 \pm 0.4) 1 / \mathrm{pF}$ were extracted.

In Fig. 3 it is seen that the additional noise, $D$, is not constant at $\tau=(20 \div 500)$ ns. It varies strongly and increases at small ( $\tau=20 \mathrm{~ns})$ and large $(\tau=500 \mathrm{~ns})$ shaping times by a factor of about two in comparison with the minimal value $D \simeq 650$ electrons at $\tau=100 \mathrm{~ns}$. This indicates 
remaining notable parallel and serial noises, which originate from the Clear Pulse 4467A shaping amplifier we use (with ORTEC 570 shaping amplifier we obtained $D \simeq 700$ electrons at $\tau=500 \mathrm{~ns}$ ).

We observed good agreement between measured total ENC and the calculated one (based on the separately measured ENC components), which indicates sufficient suppression of the correlated noises in our spectrometric channel. We also conclude that there is no room for the further essential improvement of the ENC (at room temperature).

\section{Measurement of equivalent noise energy}

Scintillation counter is made of a pure CsI crystal [7] $\left(6 \times 6 \times 30 \mathrm{~cm}^{3}\right)$ wrapped by one layer of a Gore-Tex teflon of $200 \mu \mathrm{m}$ thickness and placed in a $40-\mu \mathrm{m}$ aluminized mylar container. One or several APDs are coupled to the back facet $\left(6 \times 6 \mathrm{~cm}^{2}\right)$ of the crystal with the optical grease OKEN-6262A as shown in Fig. 4(a).

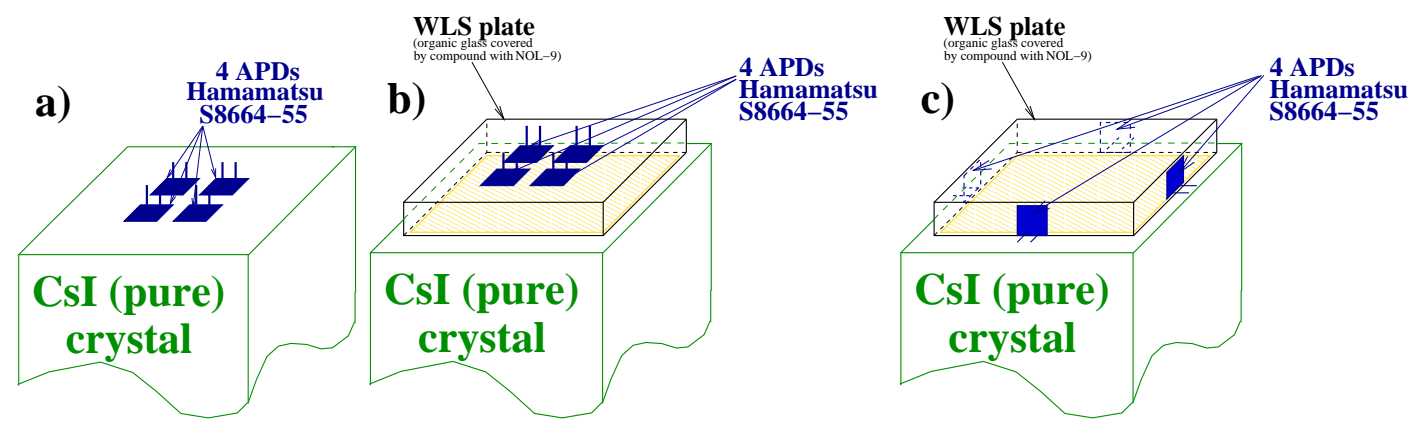

Figure 4: Scheme of the counter: (a) APDs are coupled to the pure CsI crystal through the optical grease OKEN-6262A, (b) APDs are coupled to the back side of the wavelength shifting plate with the optical grease OKEN-6262A, (c) APDs are coupled to the edge sides of the wavelength shifting plate with the optical grease OKEN-6262A.

To calibrate ADC channels in the units of deposited energy $(\mathrm{MeV})$ measurement with the signals from the cosmic muons (so called cosmic calibration) was carried out. Monte Carlo simulation of the passage of cosmic muons through the crystal shows that the most probable energy deposition (position of the cosmic peak in the amplitude spectrum) is $E_{\text {peak }}^{\mathrm{MC}}=33 \mathrm{MeV}$. The ENE in the spectrometric channel of the counter was evaluated with help of the electronic calibration as described in Section 2. The only difference is that the r.m.s. of the calibration peak was evaluated in the units of $\mathrm{MeV}$. The specific light output $(s L O)$ of the counter was evaluated as $s L O=\frac{N_{\text {cos }}}{E_{\text {peak }}^{\mathrm{M} G S_{\mathrm{APD}}}}=(26 \pm 2)$ photoelectrons $/ \mathrm{MeV} / \mathrm{cm}^{2}$, where $N_{\text {cos }}$ is number of electrons (as to the preamplifier input) corresponding to the energy deposition $E_{\text {peak }}^{\mathrm{MC}}=33 \mathrm{MeV}, G$ is APD gain, $S_{\mathrm{APD}}$ is photosensitive area of APD $\left(S_{\mathrm{APD}}=0.25 \mathrm{~cm}^{2}\right.$ for $\mathrm{S} 8664-55 \mathrm{APD}, S_{\mathrm{APD}}=1.0 \mathrm{~cm}^{2}$ for $\mathrm{S} 8664-1010$ APD).

For the counter with one S8664-1010 [S8664-55] APD the ENE was measured to be (1.54 \pm $0.15) \mathrm{MeV}[(2.3 \pm 0.2) \mathrm{MeV}]$. Although the ENC of the counter with APD S8664-1010 is larger than that with APD S8664-55, see Fig. 3, the ENE of the latter counter is bigger because its light output, determined by the photosensitive area of APD, is 4 times smaller. To decrease the equivalent noise energy several APDs $\left(N_{\mathrm{APD}}\right)$ can be coupled to one crystal, in this case the ENE is reduced 
by a factor of $1 / \sqrt{N_{\mathrm{APD}}}$. In the scheme with two S8664-1010 APDs the ENE was measured to be $(1.1 \pm 0.1) \mathrm{MeV}$. In the case of two or four S8664-55 APDs per crystal, we obtained ENE = $(1.7 \pm 0.2) \mathrm{MeV}$ or $\mathrm{ENE}=(1.2 \pm 0.1) \mathrm{MeV}$ (see also Fig. 5), respectively. Another way to decrease the ENE is to improve light output of the counter.

\section{Improvement of the light output}

To increase the light collection efficiency we studied the effects of different optical greases and thickness of reflector, Gore-Tex teflon. Three types of the silicon optical grease, OKEN-6262A, BC-630 and TSF451-50M were applied, obtained signal ratio is 1:0.95:0.85 (OKEN-6262A: BC630: TSF451-50M). In the further studies we used OKEN-6262A. It was already demonstrated that the Gore-Tex teflon has the largest reflectivity among the well-known soft reflectors even in the ultraviolet (UV) range [8]. We measured signals from the counters with the teflon of different thicknesses. It was found that the optimal thickness of teflon is $200 \mu \mathrm{m}$, its further increase improves the signal by less than $5 \%$.

The scintillation light emission spectrum of pure CsI peaks at around $320 \mathrm{~nm}$. In this UV region the quantum efficiency of the S8664 series APD is only about 30\%. Novel wavelength shifting (WLS) plates containing nanostructured organosilicon luminophores $[9,10,11]$ were developed by LumInnoTech LLC [12]. Nanostructured organosilicon luminophores (NOLs) are branched molecular structures having two types of covalently bonded via silicon atoms organic luminophores with efficient Forster energy transfer between them. Organic luminophores of the first type (energy donor) absorb the UV light, while those of the second type (energy acceptor) emit the light in the visible range. Bithiophenes are used as the energy donors and different derivatives of benzothiadiazole, emitting in green, yellow or red region, as the energy acceptors. WLS plates with NOLs allow us to shift the spectrum of the scintillation light from UV to the visible range, where the quantum efficiency of APD reaches $85 \%$. Plates with three types of NOLs were tested (NOL-9, NOL-10 and NOL-14).

The WLS plate is mounted to the back facet of the crystal without optical contact. APDs can be coupled to the plate with OKEN-6262A in two ways, to the back facet (see Fig. 4(b)), and to the edge sides of the plate (see Fig. 4(c)). The largest light output increase (by a factor of more than three) was achieved with NOL-9 luminophore, when APDs are coupled to the edge sides of the WLS plate, see Fig. 5. The ENE of counter with four S8664-55 APDs coupled to the edge sides of the NOL-9 based WLS plate was measured to be $(0.41 \pm 0.04) \mathrm{MeV}$ (at the shaping time of $50 \mathrm{~ns}$ ). The ENE with one [two] S8664-1010 APD[s] coupled to the back side of the NOL-9 based WLS plate was measured to be $(0.54 \pm 0.05) \mathrm{MeV}[(0.44 \pm 0.04) \mathrm{MeV}]$ (at the shaping time of $50 \mathrm{~ns})$.

\section{Conclusion}

Hamamatsu APDs of S8664 series were proved to be appropriate photosensitive elements for the conversion of the scintillation light from pure CsI crystals in the Belle II end cap ECL. The wavelength shifting plates with nanostructured organosilicon luminophores (NOL-9) provide large improvement of the light output. Several Hamamatsu S8664-55 APDs, coupled to the edge sides of the thick wavelength shifting plate (see Fig. 4(c)), allows us to decrease substantially the 

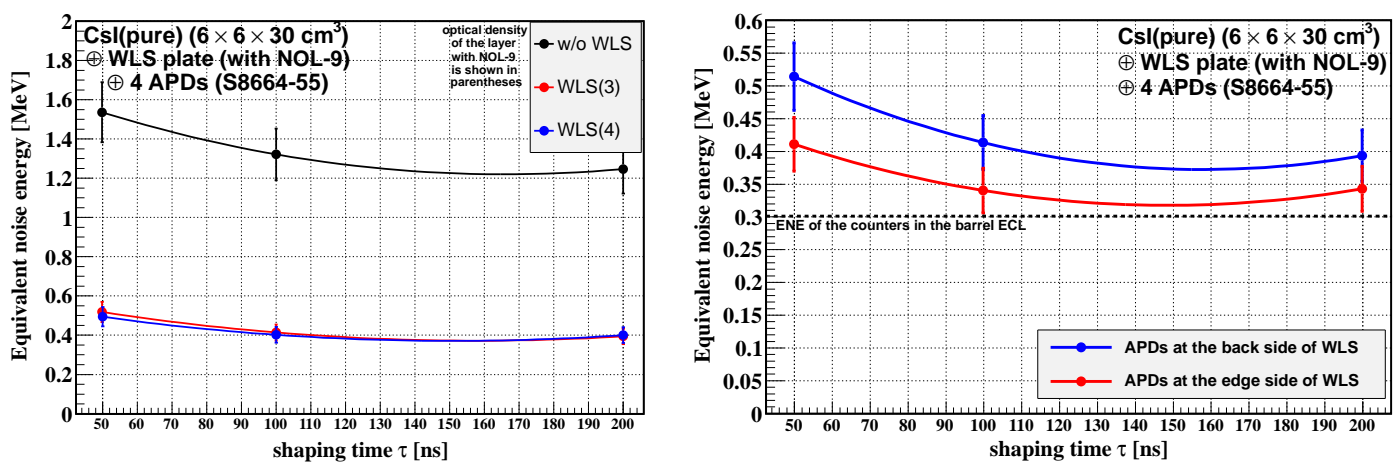

Figure 5: The ENE of the counter based on the pure CsI crystal and 4 S8664-55 APDs. APDs are coupled to the back facet of the WLS(NOL-9) plate (left), comparison of the ENEs for the counters with APDs coupled to the back facet and to the edge sides of the plate (right). Left figure also shows the ENE of the counter without WLS plate.

equivalent noise energy of the counter and provide redundancy in the signal readout. The obtained $\mathrm{ENE}=(0.41 \pm 0.04) \mathrm{MeV}$ satisfies project requirements.

\section{Acknowledgments}

O.V.B., N.M.S. and S.A.P. thank for financial support Russian Foundation for Basic Research (grant 13-03-12451).

\section{References}

[1] A. J. Bevan et al. [BaBar and Belle Collaborations], Eur. Phys. J. C 74 (2014) 3026 [arXiv:1406.6311 [hep-ex]].

[2] A. Abashian et al. [Belle Collaboration], Nucl. Instrum. Meth. Phys. Res. Sect. A 479, 117 (2002); also see detector section in: J. Brodzicka et al. [Belle Collaboration], Prog. Theor. Exp. Phys. 2012 (2012) 04D001 [arXiv:1212.5342 [hep-ex]].

[3] S. Kurokawa and E. Kikutani, Nucl. Instrum. Methods Phys. Res. Sect. A 499, 1 (2003), and other papers included in this Volume; T.Abe et al., Prog. Theor. Exp. Phys. 2013, 03 A001 (2013) and following articles up to 03A011.

[4] T. Aushev et al., arXiv:1002.5012 [hep-ex].

[5] T. Abe et al. [Belle-II Collaboration], arXiv:1011.0352 [physics.ins-det].

[6] H. Spieler, Ser. Semicond. Sci. Tech. 12 (2005) 1.

[7] Produced by Amcrys company, Kharkov, Ukraine. http://www.amcrys.com/

[8] M. Janecek, IEEE Trans. Nucl. Sci. 59.3 (2012) 490.

[9] S. A. Ponomarenko, et al., Sci. Rep. 46549 (2014).

[10] N. Surin, et al., Nucl. Instrum. Meth. A 766 (2014) 160.

[11] D. Yu. Akimov, et al., Nucl. Instrum. Meth. A 695 (2012) 403.

[12] http://www.luminnotech.com/ 